\title{
ESTADO NUTRICIONAL, CONSUMO DE ALIMENTOS ULTRA PROCESADOS Y TRASTORNO POR DÉFICIT DE LA ATENCIÓN, HIPERACTIVIDAD E IMPULSIVIDAD EN ALUMNOS DE SECUNDARIA DE LA CIUDAD DE MÉXICO
}

\author{
NUTRITIONAL STATUS, CONSUMPTION OF ULTRA-PROCESSED FOODS AND ATTENTION DEFICIT \\ DISORDER, HYPERACTIVITY AND IMPULSIVITY IN SECONDARY SCHOOL STUDENTS IN MEXICO CITY.
}

Moreno-Altamirano Laura*, Flores-Ocampo Angélica Estefanía*, Ceballos-Rasgado Marena*, García-

García Juan José *.

*Universidad Nacional Autónoma de México, Facultad de Medicina. México.

Citation: Moreno-Altamirano L., Flores-Ocampo A.E., Ceballos-Rasgado M., García-García J.J. (2021) Estado nutricional, consumo de alimentos ultra procesados y trastorno por déficit de la atención, hiperactividad e impulsividad en alumnos de secundaria de la Ciudad de México. Revista Salud Pública y Nutrición, 20 (2), 32-41.

Editor: Esteban G. Ramos Peña, Dr. CS., Universidad Autónoma de Nuevo León, Facultad de Salud Pública y Nutrición, Monterrey Nuevo León, México. Copyright: (C2021 Moreno-Altamirano L., et al. This is an open-access article distributed under the terms of Creative Commons Attribution License [CC BY 4.0], which permits unrestricted use, distribution, and reproduction in any medium, provided the original author and source are credited.

Competing interests: The authors have declared that no competing interests exist.

DOI: https://doi.org/10.29105/respyn20.2-4

Recibido: 8 de septiembre 2020; Aceptado: 13 de enero 2021

Email: garciagij@gmail.com 


\title{
ESTADO NUTRICIONAL, CONSUMO DE ALIMENTOS ULTRA PROCESADOS Y TRASTORNO POR DÉFICIT DE LA ATENCIÓN, HIPERACTIVIDAD E IMPULSIVIDAD EN ALUMNOS DE SECUNDARIA DE LA CIUDAD DE MÉXICO
}

\author{
Moreno-Altamirano Laura*, Flores-Ocampo Angélica Estefanía*, Ceballos-Rasgado Marena*, García-García \\ Juan José *. \\ * Universidad Nacional Autónoma de México, Facultad de Medicina. México.
}

\section{RESUMEN}

Introducción. El aumento de obesidad y síntomas del trastorno por déficit de atención con hiperactividad, motivó este estudio. Objetivo: Identificar estado nutricional, consumo de alimentos, trastorno por déficit de atención con hiperactividad y explorar su relación con la alimentación en alumnos de una secundaria. Material y Método: Estudio transversal realizado en todos los alumnos que contaron con aceptación para participar. Se calcularon frecuencias simples de índice de masa corporal, coeficiente de correlación de Spearman y prueba U de Mann-Whitney para explorar la relación entre alimentación y trastorno por déficit de atención con hiperactividad. El nivel de significancia fue de $5 \%(\alpha=0.05)$. Resultados: El $45.5 \%$ de alumnos tenían sobrepeso y obesidad. Entre el $18.9 \%$ y $40.5 \%$ consumían más de 3 productos ultra procesados por semana. La prevalencia de casos sospechosos del trastorno por déficit de atención con hiperactividad fue de $2.5 \%$. El consumo de golosinas (dulces) se correlacionó con el trastorno y el consumo de frituras (botanas saladas) se asoció sólo con hiperactividad. El consumo de frutas se correlacionó inversamente con hiperactividad. Conclusiones: Es necesario continuar estudiando esta asociación y enfatizar en la promoción de una alimentación saludable para prevenir el aumento de obesidad en adolescentes.

Palabras Clave: Obesidad y sobrepeso en adolescentes, productos ultra procesados, alimentación, trastornos por déficit de la atención e hiperactividad.

\section{ABSTRACT}

Introduction: Increased obesity and symptoms of attention deficit hyperactivity disorder prompted this study. Objective: Identify nutritional status, food consumption, attention deficit hyperactivity disorder, and explore their relationship to eating in secondary students. Material and method: Cross-sectional study carried out on all students who were accepted to participate. Simple body mass index frequencies, Spearman correlation coefficient, and Mann-Whitney U-test were calculated to explore the relationship between eating and attention deficit hyperactivity disorder. The significance level was $5 \%$ ( $\alpha \mathrm{x}$ 0.05). Results: $\quad 45.5 \%$ of students were overweight and obese. Between $18.9 \%$ and $40.5 \%$ consumed more than 3 ultraprocessed products per week. The prevalence of suspected cases of attention deficit hyperactivity disorder was $2.5 \%$. Consumption of sweets was correlated with the disorder and the consumption of frying (salted snacks) was associated only with hyperactivity. Fruit consumption was inversely correlate with hyperactivity. Conclusions: It is necessary to continue to study this association and emphasize the promotion of healthy eating to prevent the increase in obesity in adolescents.

Key words: Obesity and overweight in adolescents, Food-Processing Industry, ultra-processed food, eating, attention deficit disorders and hyperactivity. 


\section{Introducción}

El cambio de alimentación, en adolescentes y todos los grupos de edad, ha favorecido el aumento de la prevalencia de sobrepeso y obesidad $(\mathrm{SP}+\mathrm{O})$ en México. Entre 1988 y 2012 en los adolescentes de 12 a 19 años, el SP+O pasó de $11.1 \%$ a 34.9\%. En 2018, en este grupo de edad, fue de $38.4 \%$, en mujeres $41.4 \%$ y en hombres $35.8 \%$ (Instituto Nacional de Salud Pública INSP, 2012; 2018).

De acuerdo con informe de la OPS entre 2000 y el 2103152013 las ventas de los productos ultra procesados (UP) crecieron en México, las cuales se asocian con el sobrepeso y la obesidad. Pan American Health Organization (PAHO, 2015).

La evidencia, de múltiples autores, muestra resultados consistentes de que los UP son los principales generadores de la obesidad mundial, publicaciones referidas por la PAHO. (PAHO, 2019).

Acorde a la clasificación NOVA (El nombre que se le dio a la clasificación NOVA, se origina del destello que los astrónomos antiguos veían ante la aparición de una nueva estrella en el cielo nocturno, que en latín significa «nueva»), agrupa los alimentos por su grado de procesamiento y aporte nutricional. Los alimentos UP son "formulaciones industriales con gran cantidad de ingredientes. Se componen de sustancias que no tienen uso culinario, que son sintetizadas a partir de constituyentes de alimentos. Asimismo, contienen varios aditivos para modificar su color, sabor o textura final (PAHO, 2015). Algunos de estos aditivos son inocuos, de otros se conocen diversos efectos en la salud, sobre todo cuando se consumen grandes porciones y algunos más, están en discusión (Aditivos alimentarios; Matthiesen, Fagt, Biltoft-Jensen, Beck, Ovesen, 2003).

Por ejemplo, los azúcares, el glutamato monosódico (GMS) y el ácido guanílico pueden desviar mecanismos en el sistema digestivo y el cerebro asociados a la señal para saciar el apetito y, por lo tanto, causar sobreconsumo, obesidad y afectar negativamente los hábitos alimentarios (Aditivos alimentarios). La mayoría de los alimentos y bebidas UP, incluso, muchos productos "light" están endulzados con jarabe de maíz de alta fructosa que tiene un índice glucémico muy alto por lo que se eleva en forma inmediata el nivel de azúcar en sangre (Johnson, et al., 2007).

Existen evidencias de que el GMS y todos los glutamatos, el mono-potásico, el cálcico, amónico, y magnésico, puede contribuir a la fisiopatología del trastorno por déficit de la atención (TDAH). A este compuesto, se le llama también proteína vegetal, fermentos autolizados, proteína de soya, proteína o vegetales hidrolizados, soya texturizada, caseinatos, saborizantes naturales, etc (Maltezos et al., 2014).

Se ha documentado que el GMS genera genotoxicidad en linfocitos humanos debido a que aumenta el daño oxidativo celular y conlleva alteraciones en el DNA (Lau, McLean, Williams y Howard, 2006). El GMS está contraindicado en personas con TDAH, trastorno bipolar, Alzheimer, Parkinson, epilepsia y esquizofrenia (Nigg y Holton, 2014). Se ha recomendado que los pacientes con TDAH eliminen de su dieta colorantes, saborizantes y endulzantes artificiales, GMS y monopotásico, benzoato de sodio, carragenos (Nigg y Holton, 2014; Kaplan, McNicol, Conte y Moghadam 1989). El GMS se utiliza para acentuar el sabor de los UP que se consumen sobre todo por niños y adolescentes. En los hogares es utilizado ampliamente en pastillas de consomé, salsas, sopas, condimentos industriales, etc (Aditivos alimentarios).

A casi todos los UP se les adicionan, entre otros aditivos, colorantes artificiales como rojo 40, amarillo 5 (tartrazina o tartracina), amarillo 6, rojo cochinilla, carmoisina y ponceau $4 \mathrm{R}$, los cuales están asociados a incremento de los síntomas de TDAH. Otros aditivos que se utilizan en la mayoría de los UP son el ácido fosfórico y almidón modificado también asociados con TDAH (Aditivos alimentarios).

El TDAH es uno de los principales trastornos neuropsiquiátricos en la infancia. Se caracteriza por capacidad de atención disminuida, hiperactividad e impulsividad, que generan deterioro en el funcionamiento escolar y general (American Psychiatric Association. DSM-IV: DSM-IV, 1994).

El objetivo de este estudio fue Identificar el estado nutricional medido a través del Índice de masa corporal (IMC), el consumo de alimentos y el trastorno por déficit de atención con hiperactividad (TDAH) y sus componentes y explorar su relación 
con la alimentación en alumnos de una secundaria de la Ciudad de México.

\section{Material y Método}

Diseño y población.

Se realizó en el año 2018, un estudio transversal en, prácticamente, la totalidad del alumnado (6 grupos de cada uno de los 3 grados), del turno matutino de una escuela secundaria pública de la Ciudad de México que aceptó participar, con el conocimiento y autorización de sus padres. La población estuvo conformada por 473 estudiantes, 241 mujeres y 232 hombres, de quienes se obtuvieron medidas antropométricas. El cuestionario de alimentos fue contestado por 460 alumnos, en tanto que el de TDAH, lo fue por 472 alumnos, de los cuales 237 $(50.1 \%)$ fueron mujeres y $235(49.9 \%)$ hombres.

\section{Variables y proceso de obtención de datos. Consumo de alimentos.}

Se aplicó un cuestionario de "Frecuencia de Consumo de Alimentos" elaborado y validado por el profesorado participante en este estudio. El grupo académico, conformado por epidemiólogos y nutriólogos, contempló la validez de apariencia, de contenido y de expresión del instrumento, conformado por 25 preguntas sobre ingesta de alimentos procesados o caseros y mínimamente procesados y productos ultra procesados (NOVA).

Se calculó la media del consumo de alimentos y bebidas, agrupados acorde con la clasificación NOVA (PAHO, 2015).

\section{TDAH.}

Se utilizó el "Cuestionario para Escolares y Adolescentes Latinoamericanos con Trastorno por Déficit de Atención con Hiperactividad" (CEALTDAH, 2009), con 28 reactivos (15 evalúan inatención, 7 hiperactividad y 6 impulsividad). Se califica en una escala tipo Likert del 0 al 3 con una puntuación máxima de 84 puntos. Se consideraron como casos sospechosos a los alumnos que obtuvieron 51 puntos o más, con base en 17 criterios. El punto de corte correspondió al percentil 97.5 de la distribución de valores. Para los componentes de TDAH, dicho percentil se ubicó en 30 puntos para inatención, 14 puntos para hiperactividad, y 12 puntos para impulsividad.
Índice de masa corporal (IMC).

Se midió la estatura y el peso de los alumnos de secundaria (con una báscula Tanita, y estadímetro), y se calculó el IMC $(\mathrm{kg} / \mathrm{m} 2)$. Se utilizaron las Tablas de IMC y tablas de IMC para la edad, de niños(as) y adolescentes de 5 a 18 años de edad. Se consideró la existencia de sobrepeso cuando el IMC para la edad se encontraba más de una desviación estándar por encima de la mediana establecida en los patrones de crecimiento infantil de la OMS, y obesidad cuando era mayor que dos desviaciones estándar por encima de la mediana establecida en los patrones de crecimiento infantil de la OMS. (OMS, 2020 c). (Tabla I)

Tabla 1. Puntos internacionales de corte de IMC para sobrepeso y obesidad en niños, por sexo, de 11 a 16 años, correspondientes a un IMC de 25 y de 30 a los 18 años

\begin{tabular}{ccccc}
\hline \multirow{2}{*}{ Edad } & \multicolumn{2}{c}{ IMC $25 \mathrm{~kg} / \mathrm{m}^{2}$} & \multicolumn{2}{c}{$\mathrm{IMC} 30 \mathrm{~kg} / \mathrm{m}^{2}$} \\
& Hombres & Mujeres & Hombres & Mujeres \\
\hline 11.0 & 20.6 & 20.7 & 25.1 & 25.4 \\
11.5 & 20.9 & 21.2 & 25.6 & 26.1 \\
12.0 & 21.2 & 21.7 & 26.0 & 26.7 \\
12.5 & 21.6 & 22.1 & 26.4 & 27.2 \\
13.0 & 21.9 & 22.6 & 26.8 & 27.8 \\
13.5 & 22.3 & 23.0 & 27.3 & 28.2 \\
14.0 & 22.6 & 23.3 & 27.6 & 28.6 \\
14.5 & 23.0 & 23.7 & 28.0 & 28.9 \\
15.0 & 23.3 & 23.9 & 28.3 & 29.1 \\
15.5 & 23.6 & 24.2 & 28.6 & 29.3 \\
16.0 & 23.9 & 24.4 & 28.9 & 29.4 \\
\hline
\end{tabular}

Fuente: Adaptado de: Kaufer H. M., Toussaint G. (2008) Indicadores antropométricos para evaluar sobrepeso y obesidad en pediatría. Boletín Médico del Hospital Infantil de México. (65), 502-518

Un grupo de estudiantes de Medicina fue capacitado para la aplicación de los instrumentos, para realizar la somatometría y para calcular el IMC (este se verificó calculándolo de manera automatizada en Excel).

\section{Análisis estadístico}

Se obtuvo la prevalencia de sobrepeso y obesidad y se calculó la media del consumo de alimentos y bebidas. Debido a que las variables antropométricas no siguieron una distribución semejante a la normal, 
se utilizó la mediana y otros percentiles para describirlas, se realizó la prueba U de Mann-Whitney para comparar dichas distribuciones, así como las puntuaciones de TDAH, según sexo, y se obtuvo el coeficiente de correlación de Spearman entre la puntuación del cuestionario de TDAH (51 puntos y más) y edad, IMC, y la frecuencia de consumo de alimentos. Se utilizó un nivel de significancia de 5\% $(\alpha=0.05)$. Se utilizó el programa SPSS (versión 25).

\section{Consideraciones éticas}

El proyecto fue revisado por las Comisiones de Ética e Investigación y registrado en la División de Investigación de la Facultad de Medicina UNAM como parte del protocolo FM/DI/117/2017. 20172020 .

Todos los participantes tuvieron conocimiento de la finalidad de la investigación, su participación fue voluntaria y se contó con la autorización de sus padres para que ellos participaran y para que sus datos pudieran ser utilizados para su publicación, reproducción y divulgación en soporte de papel e Internet.

\section{Resultados}

Datos generales. La edad promedio fue de 13.10 años \pm 0.94 (DS) para las mujeres y de $13.19 \pm 0.98$ (DS) para los hombres, en tanto que la mediana fue de 13 para ambos.

\section{Medidas antropométricas.}

La media de la talla fue de $1.54 \mathrm{~m} \pm 0.06$ (DS) para mujeres y $1.60 \mathrm{~m} \pm 0.09$ (DS) para hombres, mientras que la mediana fue de $1.54 \mathrm{~m}$ y $1.61 \mathrm{~m}$, respectivamente; el peso promedio de las mujeres fue de $53.7 \mathrm{~kg} \pm 13$ (DS) y el de los hombres de $56.2 \mathrm{~kg}$ \pm 14.4 (DS). La mediana, en cambio, fue de 51 y 54 $\mathrm{kg}$, respectivamente.

La media del IMC fue de $22.1 \mathrm{~kg} / \mathrm{m} 2 \pm 4.7$ (DS), y de $21.3 \mathrm{~kg} / \mathrm{m} 2 \pm 4.5$ (DS), para mujeres y hombres, respectivamente. Por otro lado, la mediana fue de 21.1 y de 20.1 .

Se encontraron diferencias estadísticamente significativas en la distribución del peso y la talla, según sexo (Prueba U de Mann-Whitney, p < 0.05 y $\mathrm{p}<0.01$, respectivamente), pero no en la edad y el IMC.
El 2.5\% del alumnado presentó bajo peso. Por otro lado, se observó SP en el $23.3 \%$ y O en el $22.2 \%$ de los. El SP fue más frecuente en mujeres mientras la obesidad fue mayor en hombres. (Tabla 2).

Tabla 2. Estado nutricional por sexo en alumnos de secundaria

\begin{tabular}{lcccccc}
\hline Estado & \multicolumn{2}{c}{ Mujeres } & \multicolumn{2}{c}{ Hombres } & \multicolumn{2}{c}{ Total } \\
nutricional (IMC) & No. & $\%$ & No. & $\%$ & No. & $\%$ \\
\hline Bajo peso severo & 1 & 0.4 & 1 & 0.4 & 2 & 0.4 \\
Bajo peso & 3 & 1.2 & 7 & 3 & 10 & 2.1 \\
Normal & 128 & 53.1 & 118 & 50.9 & 246 & 52 \\
Sobrepeso & 61 & 25.3 & 49 & 21.1 & 110 & 23.3 \\
Obesidad & 48 & 19.9 & 57 & 24.6 & 105 & 22.2 \\
Total & 271 & 100 & 232 & 100 & 473 & 100 \\
\hline
\end{tabular}

Fuente: Encuesta

Productos ultra procesados (UP).

Consumo diario.

El $68.9 \%$ de los alumnos consumió uno o dos golosinas (dulces), el $29.6 \%$ tres o más. El $81.8 \%$ refirió consumir de 1 a 2 frituras (botanas saladas), mientras que el $16.4 \%$ ingirió tres o más bolsas. El $74.4 \%$ de los alumnos beben entre 1 y 2 lácteos de sabor, el $22.8 \%$ consumen 3 o más.

\section{Consumo semanal.}

El 79.5\% de los alumnos consumen comida rápida de 1 a 2 veces, casi el 19\% los hace tres o más. El 62.7\% refirió consumir una sopa instantánea y el $16.4 \%$ consume más de tres. El $58.0 \%$ de los alumnos comen carne procesada entre 1 y 2 días. El $40.5 \%$ lo hace 3 o más. El $57.3 \%$ consume cereales dulces de una a dos veces, el $39.6 \%$ tres o más días. El $73 \%$ de los alumnos consumen pan de caja de una a dos veces, el $27.4 \%$ tres o más días. El $71.6 \%$ usan aderezos una o dos veces, el $23.7 \%$, en 3 o más ocasiones. El 35.5\% de los alumnos beben 3 o más refrescos y consumen la misma cantidad de jugos envasados (Tabla 3 ). 
Tabla 3. Consumo de alimentos y bebidas ultra procesados en alumnos de secundaria

\begin{tabular}{lc}
\hline Alimentos y bebidas ultra procesadas *3 o más & $\%$ \\
porciones & 40.5 \\
Carne procesada a la semana & 39.6 \\
Cereales dulces a la semana & 29.6 \\
Golosinas (dulces, chocolates, helados, pastelillos) al día & 27.4 \\
Pan de caja a la semana & 23.7 \\
Aderezos a la semana & 18.9 \\
Comida rápida a la semana & 16.4 \\
Frituras al día & 16.4 \\
Sopas instantáneas a la semana & \\
& \\
Bebida no láctea azucarada a la semana: & 35.5 \\
Refrescos & 35 \\
Jugos & 22.8 \\
Bebidas lácteas azucaradas al día & \\
\hline Fuente: Encuesta & \\
*Todos los alumnos consumen por lo menos un día a la semana todos los UP
\end{tabular}

\section{Alimentos mínimamente procesados y procesados.}

Consumo diario.

El $52.2 \%$ de los adolescentes consumen entre 1 y 2 frutas, el resto 3 o más. El $51.4 \%$ de los estudiantes ingieren 4 o más verduras. El $47 \%$ de los alumnos beben más de cinco vasos de agua natural, el resto 4 o menos. El 56\% de ellos beben 3 o más vasos de agua de fruta natural con azúcar.

Consumo semanal.

El $42.7 \%$ de los alumnos comen 2 o menos porciones de leguminosas. El 69.3\% de ellos consumen 3 o más porciones de cereales, en particular maíz (tortillas). El $55.3 \%$ corresponde a arroz, el $26.9 \%$ a pasta (trigo) y el $26.9 \%$ a antojitos. El consumo de 3 o más porciones de cárnicos fue: $40 \%$ pollo, $35 \%$ res y $13 \%$ pescado (Tabla 4).

\begin{tabular}{|c|c|}
\hline Alimentos y bebidas procesadas o mínimamente procesadas: porciones/vasos al día & $\%$ \\
\hline Agua de frutas con azúcar (3 o más) & 56 \\
\hline Verduras (4 o más) & 51.4 \\
\hline Agua (5 o más) & 47 \\
\hline Frutas (3 o más) & 46.8 \\
\hline Alimentos procesados o mínimamente procesados* 3 o más porciones/semana & $\%$ \\
\hline Tortillas de maíz & 69.3 \\
\hline Leguminosas & 55.8 \\
\hline Cereales (Arroz o pasta) & 55.3 \\
\hline Huevo & 44 \\
\hline Antojitos & 26.9 \\
\hline Carnes no procesadas: & $\%$ \\
\hline Pollo & 40 \\
\hline Res & 35 \\
\hline Pescado & 13 \\
\hline
\end{tabular}

Trastornos por déficit de atención e hiperactividad (TDAH).

La prevalencia global de casos sospechosos de TDA fue de $2.5 \%$. Al desagregar los componentes de TDA, se calificaron 13 personas con inatención, 17 con hiperactividad y 18 con impulsividad. Al analizar la distribución de cada uno de dichos componentes según sexo con la prueba U de MannWhitney, se observaron diferencias estadísticamente significativas en las puntuaciones de hiperactividad entre hombres y mujeres, $(\mathrm{p}<0.001)$, pero no en las de inatención e impulsividad (Tabla 5).

\begin{tabular}{|c|c|c|c|c|c|c|c|c|c|c|c|c|}
\hline \multirow[b]{2}{*}{ Percentil } & \multicolumn{3}{|c|}{ Inatención } & \multicolumn{3}{|c|}{ Hiperactividad } & \multicolumn{3}{|c|}{ Impulsividad } & \multicolumn{3}{|c|}{ TDAH } \\
\hline & $\mathrm{H}$ & M & Global & $\mathrm{H}$ & M & Global & $\mathrm{H}$ & $\mathrm{M}$ & Global & $\mathrm{H}$ & M & Global \\
\hline 2.5 & 3 & 2 & 2 & 1 & 0 & 0 & 0 & 0 & 0 & 4.9 & 4 & 4 \\
\hline 25 & 8 & 8 & 8 & 3 & 2.5 & 3 & 2 & 2 & 2 & 15 & 13 & 14 \\
\hline 50 & 12 & 11 & 12 & 5 & 4 & 5 & 4 & 4 & 4 & 21 & 20 & 20 \\
\hline 75 & 16 & 16.5 & 16 & 8 & 6 & 7 & 7 & 12 & 7 & 30 & 28.5 & 29.3 \\
\hline 97.5 & 28.2 & 31 & 30 & 15 & 14.1 & 14 & 13 & 12 & 12 & 53 & 52.1 & 52.2 \\
\hline Mínimo & 0 & 0 & 0 & 0 & 0 & 0 & 0 & 0 & 0 & 1 & 2 & 1 \\
\hline Máximo & 43 & 42 & 43 & 21 & 20 & 21 & 18 & 14 & 18 & 82 & 74 & 82 \\
\hline
\end{tabular}

\section{Alimentación y TDAH.}

El consumo diario de golosinas se correlacionó con cada uno de los componentes de TDA, así como con la evaluación global. La cantidad de frituras consumidas al día se asoció estadísticamente significativa sólo con hiperactividad. Aunque débil, el consumo de frutas se correlacionó inversamente con hiperactividad. (Tabla 6).

Tabla 6. Componentes del TDAH y alimentos ultra procesados

\begin{tabular}{lccc}
\hline $\begin{array}{c}\text { Trastorno de déficit de } \\
\text { atención }\end{array}$ & $\begin{array}{c}\text { Consumo diario de } \\
\text { alimentos }\end{array}$ & $\begin{array}{c}\text { Correlación de } \\
\text { Spearman }\end{array}$ & Valor de p \\
\hline Inatención & Golosinas & 0.178 & $<0.05$ \\
Hiperactividad & Fruta & -0.82 & $<0.05$ \\
& Golosinas & 0.164 & $<0.01$ \\
Impulsividad & Frituras & 0.114 & $<0.05$ \\
Evaluación global & Golosinas & 0.159 & $<0.05$ \\
\hline & Golosinas & 0.195 & $<0.01$ \\
\hline
\end{tabular}

TDAH: Trastorno por déficit de atención e hiperactividad. 


\section{Discusión}

En este estudio fue posible corroborar que el $\mathrm{SP}+\mathrm{O}$ es un problema que se debe atender urgentemente. Las cifras encontradas en este estudio (45.5\%) fueron superiores a las reportadas por ENSANUT 2018 en adolescentes de 12 a 19 años (38.4\%) (INSP, 2018). Asimismo, se infiere que la alimentación de los adolescentes estudiados es de alta densidad energética ya que gran parte de ellos señalaron consumir entre 1 y 2 presentaciones de UP, lo cual supera por mucho las recomendaciones de consumo de azúcares añadidos, grasas y sal por día. En porciones de 15 gramos es posible encontrar hasta 2 cucharadas de azúcar, $25 \%$ del consumo máximo por día recomendado por la OMS (OMS, 2015 a). Además, contienen diversos aditivos como GMS, colorantes, benzoato de sodio, ácido fosfórico, ácido guanílico, y almidón modificado, entre otros (PAHO, 2019).

Algunas golosinas contienen plomo, el que se ha relacionado con hiperactividad y conducta antisocial (Azcona, Ramírez y Vicente, 2015; Liu, Liu et al., 2014). La mayoría de los alumnos ingieren 2 paquetes de frituras por día, en los que el contenido de azúcar oscila entre el $47 \%$ y el 62\% (PROFECO, 2018). Asimismo, una porción de $30 \mathrm{~g}$ de cereal empaquetado contiene un tercio de la cantidad máxima de azúcar que puede ingerir un adolescente en un día, más de la mitad de los alumnos los consumen dos veces a la semana (PROFECO, 2011). El pan de caja, los aderezos y las sopas instantáneas, también contienen grandes cantidades de azúcares, el $80 \%$ de los estudiantes los comen por lo menos una vez a la semana. Los refrescos, los jugos y las leches saborizadas contienen además jarabe de maíz de alta fructosa y otros tipos de azúcares añadidos, el $88 \%$ de los alumnos beben entre 1 a 4 refrescos y jugos envasados por semana y más del $90 \%$ de ellos consumen de 1 a 4 productos lácteos saborizados por día (Armenteros, 2018; Pérez-Herrera y Cruz-López, 2019).

La mayoría de los jugos contienen azúcares añadidos como fructosa o sacarosa, y se asocian, por tanto, a un mayor riesgo de desarrollar sobrepeso y obesidad y enfermedades metabólicas (Cabada, 2016). Los refrescos, además, contienen aditivos químicos, como ácido fosfórico, cuyos efectos están relacionados con hiperactividad y otros problemas de salud (Magaña, 2019; Aditivos alimentarios). Los embutidos y carnes frías, que casi la mitad de los alumnos estudiados (40.5\%) refirieron consumir más de tres veces a la semana, contienen almidón modificado, asociado con TDAH (PROFECO, 2010) y tienen gran cantidad de aditivos; en el 2015 la OMS los clasificó dentro del Grupo 1, cancerígeno para los seres humanos (cáncer de colon, recto, páncreas y próstata) (OMS, 2015 b). El alto consumo de comida rápida y de sopas instantáneas orienta a suponer que varias de las comidas de los alumnos son reemplazadas por UP, ya que el $79.5 \%$ de los alumnos consumen comida rápida de 1 a 2 veces a la semana. Asimismo, el $62.7 \%$ refirió consumir una sopa instantánea al día y el $16.4 \%$ dijo consumir más de tres. Las sopas instantáneas están compuestas hasta por 36 ingredientes de los cuales tres son diferentes tipos de azúcares, siete son diferentes tipos de sales, GMS y ácido guanílico (Cabada, 2013), aditivos asociados con obesidad y TDAH. Los aderezos, consumidos por el $71.6 \%$ de los alumnos, contienen, entre otros aditivos, tartrazina que también se ha asociado a TDAH; en Europa, a partir de 2009 los alimentos que contengan este colorante deben contar con la siguiente leyenda "Puede tener efectos negativos sobre la actividad y la atención" (Aditivos alimentarios; PROFECO, 2014).

En la ENSANUT 2018 se reportó que más del 80\% de la población de todas las edades consume bebidas azucaradas y más de la mitad de los niños y adolescentes consumen botanas, dulces y postres frecuentemente (INSP, 2018).

Por otro lado, fue posible observar que los adolescentes tienen una alimentación deficiente en nutrimentos esenciales. A partir del consumo de diversos alimentos reportado por los alumnos, se infiere que no logran cubrir las necesidades de diversos nutrimentos. Por ejemplo, el consumo de frutas y verduras recomendado es de 3 frutas y 4 verduras al día (Bonvecchio et al., 2015), es menor al referido por más de la mitad de los alumnos lo cual, no logra cubrir las necesidades de vitaminas y minerales $(\mathrm{C}, \mathrm{D}$, calcio, hierro, potasio, magnesio, etc.) (Tabla III). Asimismo, la recomendación para el consumo de alimentos de origen animal (AOA) es de 3.5 porciones al día, el reportado por los alumnos es muy bajo para cubrir los requerimientos de hierro

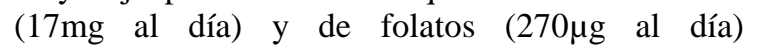
(Bonvecchio, 2015) (Tabla III). 
En este estudio se identificaron 13 alumnos (2.5\%) como posibles casos con TDAH. Mientras que en el mundo se ha reportado que la prevalencia es de 5.29\% (Polanczyk et al., 2007; Willcutt, 2012). Vale la pena señalar que los niños con TDAH que no son tratados tienen mayor riesgo de presentar accidentes y heridas (Ulloa, Sauceda, Ortiz y Sánchez, 2006; Leibson y Long, 2003), Además manifiestan un pobre desarrollo académico y alto abandono de la escuela. Este trastorno puede persistir durante la vida adulta por lo que las relaciones familiares y maritales se ven afectadas (Sauceda, Albores, Capece, Landeros y Martínez, 2007), de ahí la importancia de identificarlos y tratarlos oportunamente.

Debido a lo señalado líneas arriba fue que en este estudio transversal se decidió estudiar la asociación entre UP que contienen aditivos como los colorantes, el GMS, etc. Si bien se puede observar una posible asociación entre golosinas y TDAH y entre la cantidad de frituras con hiperactividad, debido a que no se realizó un estudio longitudinal no es posible inferir causalidad (Tabla VI).

En contraparte, se observó una leve correlación inversa entre el consumo de frutas y la hiperactividad. Al respecto, vale la pena destacar que menos de la mitad de los alumnos (46.8\%) mostró un consumo adecuado de frutas (Tabla IV). Por ejemplo, en este estudio se observó que solamente alrededor de la mitad de los alumnos consumen cantidades adecuadas de verduras y leguminosas (se recomienda consumir de 1 a 2 porciones al día). Se ha documentado que en México cada vez se consume menor cantidad de leguminosas, misma que es sabido que cuentan con alto contenido de proteínas, carbohidratos, fibra, vitaminas y minerales como hierro, calcio y vitamina $\mathrm{B}$, que contribuyen a cubrir las necesidades de folatos y de cereales, los cuales contienen vitaminas B, hierro, etc (MorenoAltamirano et al, 2014; Moreno-Altamirano et al, 2015).

Sin embargo, el consumo de cereales en este estudio fue adecuado en la mayoría de los alumnos (se recomienda entre 7 y 8 porciones al día), así como la ingesta de agua natural ( 3 a 8 vasos al día). Por otro lado, la cantidad de agua de frutas con azúcar añadido que los adolescentes señalaron beber fue muy alta (Bonvecchio, 2015).
No obstante, la asociación de los aditivos alimentarios y sus posibles efectos en la salud aún es controversial. Es claro que los componentes de la alimentación no son los únicos factores involucrados en la causalidad de TDAH, y la naturaleza de un estudio transversal como este sólo permite establecer asociaciones estadísticas que son susceptibles de ser abordadas con en otro tipo de diseños. Finalmente, se recomienda realizar acciones de detección temprana de este trastorno. En el presente trabajo no se pretendió realizar el diagnóstico como tal de TDAH, no obstante, se recomendó la atención especializada en los jóvenes que tuvieron datos sugestivos del mismo.

\section{Conclusiones}

Es fundamental asumir cabalmente el compromiso con el "Plan de Acción quinquenal para la prevención de la obesidad en la niñez y la adolescencia 2014-2020" (OPS 2014). El cual propone las medidas que se deben adoptar al respecto (OPS-OMS, 2014). El logro de sus objetivos requiere un enfoque multisectorial que se centre en transformar el ambiente obesogénico actual en oportunidades para promover un consumo mayor de alimentos nutritivos. Ya que sustituir estos por productos UP de alta densidad energética y ricos en aditivos, en varios estudios, se ha asociado con el incremento de la obesidad, de los síntomas del TDAH y de otros problemas de salud. En ese sentido, es necesario organizar actividades dirigidas a los padres de familia, profesores y a los alumnos de todos los niveles, para promover una alimentación saludable.

\section{Agradecimientos}

Los investigadores extendemos nuestro agradecimiento por el trabajo a los estudiantes del Grupo 2208 generación 2018-2019 de la Facultad de Medicina de la UNAM: Aguilar Saucedo Nancy Guadalupe, Alcántara Téllez Edwin Raúl, Alducin Arellano Ángel, Álvarez López Adrián, Arias Vega Gabriela Itzel, Castellanos Santiago Luis Guillermo, Castillo Reséndiz David Noé, Cornelio Cayetano Leticia, De la Cruz Vélez Arlet, Elizarrarás Herrera Lady Diana, García López Abril Itzel, García Vargas Irma Berenice, Gómez De La Peña Leticia Lore, Gutiérrez Cruz Anahí, Gutiérrez Fernández Josué Noel, Granados García Ana Laura, Jiménez Ramírez Ana Lilia, Mata Aguilera Xcaret, Medina Aguilar Fernando Miguel, Medina Galindo Nahomi, Moreno 
Ávalos Luis Enrique, Ramírez Luis Edwin, Reyna Gutiérrez Ana Mitzi, Rodríguez Zaragoza Ariel Ricardo, Sánchez Zuccolotto Anel Cecilia, Santamaría Acevedo Daniela, Tenorio Robles Michelle, Torres Santamaría Andrea, Villantes Gómez Abraham.

\section{Bibliografía}

Aditivos alimentarios. Recuperado de https://www.aditivos-alimentarios.com

American Psychiatric Association. (APA, 1994) DSM-IV: Diagnostic and Statistical Manual of Mental Disorders.

Armenteros T. (2018) Radiografía de Leche Kids, crecimiento 1 a 3 años, de Alpura. El Poder del Consumidor. Recuperado de https://elpoderdelconsumidor.org/2018/06/radio grafia-de-leche-kids-crecimiento-1-a-3-anos-dealpura/

Azcona M.I., Ramirez R. y Vicente G. (2015) Efectos Tóxicos del Plomo. Revista de especialidades médico-quirúrgicas, (20), 72-77.

Bonvecchio A. et al. (2015) Guías Alimentarias y de Actividad Física en Contexto de Sobrepeso y Obesidad en la Población Mexicana. CONACYT, (1), 52-54.

Cabada X. (2013) Radiografía de Sopa Instantánea Maruchan. El Poder del Consumidor. Recuperado https://elpoderdelconsumidor.org/2013/03/radio grafia-de-sopa-instantanea-maruchan-sabor-apollo/

Cabada X. (2016) Radiografía de Jumex Fresco Único Jugo Verde (1 litro). El Poder del Consumidor. Recuperado de https://elpoderdelconsumidor.org/2016/09/radio grafia-de-jumex-fresco-unico-jugo-verde-1-litro/

Comité Internacional para el Desarrollo y Estudio del Cuestionario para Escolares y Adolescentes Latinoamericanos con Trastorno por Déficit de Atención con Hiperactividad (CEAL-TDAH). (2009) Cuestionario para escolares y adolescentes latinoamericanos con Trastorno por
Déficit de Atención con Hiperactividad (CEALTDAH). Construcción, descripción del instrumento y datos sociodemográficos y clínicos de la muestra. Salud Mental, (32), 55-62.

Instituto Nacional de Salud Pública. (INSP, 2012) Encuesta Nacional de Salud y Nutrición - 2012. Resultados Nacionales. Secretaria de Salud México.

Instituto Nacional de Salud Pública. (INSP, 2018) Encuesta Nacional de Salud y Nutrición 2018. Presentación de Resultados. INEGI-Instituto Nacional de Salud Pública-Secretaría de Salud.

Johnson R. et al. (2007) Potential role of sugar (fructose) in the epidemic of hypertension, obesity and the metabolic syndrome, diabetes, kidney disease, and cardiovascular disease. The American Journal of Clinical Nutrition, (86), 899-906. DOI: 10.1093/ajcn/86.4.899

Kaplan B.J., McNicol J., Conte R.A. y Moghadam H.K. (1989) Dietary replacement in preschoolaged hyperactive boys. Pediatrics. (83), 7-17.

Kaufer H. M., Toussaint G. (2008) Indicadores antropométricos para evaluar sobrepeso y obesidad en pediatría. Boletín Médico del Hospital Infantil de México. (65), 502-518.

Lau K., McLean W.G., Williams D.P. y Howard C.V. (2006) Synergistic interactions between commonly used food additives in a developmental neurotoxicity test. Toxicological Sciences, (90), 178-187. DOI: $\underline{10.1093 / \text { toxsci/kfj073 }}$

Leibson C.L. y Long K.H. (2003) Economic implications of attention-deficit hyperactivity disorder for healthcare systems. Pharmacoeconomics, (21), 1239-1262. DOI: $\underline{10.2165 / 00019053-200321170-00002}$

Liu J. et al. (2014) Blood Lead Concentrations and Children's Behavioral and Emotional Problems: A Cohort Study. JAMA Pediatrics, 168(8), $737-$ 745 .

Magaña P. (2019) Radiografía de Coca-Cola Cherry sabor cereza. El Poder del Consumidor. 
Recuperado de https://elpoderdelconsumidor.org/2019/07/radio grafia-de-coca-cola-cherry-sabor-cereza/

Maltezos S. et al. (2014) Glutamate/glutamine and neuronal integrity in adults with ADHD: a proton MRS study. Translational Psychiatry, (18). doi: $\underline{10.1038 / \text { tp.2014.11 }}$

Matthiesen J., Fagt S., Biltoft-Jensen A., Beck A.M. y Ovesen L. (2003) Size makes a difference. Public Health Nutrition, (6), 65-72. DOI: 10.1079/PHN2002361

Miller E.M., Pomerleau F., Huettl P., Gerhardt G.A. y Glaser P.E. (2014) Aberrant glutamate signaling in the pre-frontal cortex and striatum of the spontaneously hypertensive rat model of attention-deficit/hyperactivity disorder. Psychopharmacology, (231), 3019-3029. DOI: 10.1007/s00213-014-3479-4

Moreno-Altamirano L. et al. (2014) La transición alimentaria y la doble carga de malnutrición: cambios en los patrones alimentarios de 1961 a 2009 en el contexto socioeconómico mexicano. Archivos Latinoamericanos de Nutrición, (64), 231-240.

Moreno-Altamirano L. et al. (2015) Diabetes tipo 2 y patrones de alimentación de 1961 a 2009: algunos de sus determinantes sociales en México. Gaceta Médica de México, (151), 354-368.

Nigg J.T. y Holton K. (2014) Restriction and Elimination Diets in ADHD Treatment. Child and Adolescent Psychiatric Clinics of North America, (23), 937-953. doi: $\underline{10.1016 / \mathrm{j} . c h c .2014 .05 .010}$

Oliva O.H. y Fragoso S. (2015) Consumo de comida rápida y obesidad, el poder de la buena alimentación. Revista Iberoamericana para la Investigación y el Desarrollo Educativo, (4), 176-199.

Organización Mundial de la Salud. (OMS, 2015 a) WHO calls on countries to reduce sugars intake among adults and children. Recuperado de https://www.who.int/news/item/04-03-2015- who-calls-on-countries-to-reduce-sugars-intakeamong-adults-and-children

Organización Mundial de la Salud. (OMS, 2015 b) Carcinogenicidad del consumo de carne roja y de la carne procesada. Recuperado de https://www.who.int/features/qa/cancer-redmeat/es/

Organización Mundial de la Salud (OMS, 2020 c) Obesidad y sobrepeso. Recuperado de https://www.who.int/es/news-room/factsheets/detail/obesity-and-overweight

Organización Panamericana de la SaludOrganización Mundial de la Salud. (OPS-OMS, 2014) Plan de acción para la prevención de la obesidad en la niñez y la adolescencia.

Pan American Health Organization. (PAHO, 2015) Ultra-processed food and drink products in Latin America: Trends, impact on obesity, policy implications.

Pan American Health Organization. (PAHO, 2019) Alimentos y bebidas ultra procesados en América Latina: ventas, fuentes, perfiles de nutrientes e implicaciones normativas. Recuperado de https://iris.paho.org/bitstream/handle/10665.2/51 523/9789275320327_spa.pdf?sequence $=1 \&$ is All owed=y

Pérez-Herrera A. y Cruz-López M. (2019) Situación actual de la obesidad infantil en México. Nutrición Hospitalaria, (36). doi: http://dx.doi.org/10.20960/nh.2116

Polanczyk G., Silva M., Lessa B., Biederman J. y Rohde L.A. (2007) The worldwide prevalence of ADHD: A systematic review and metaregression analysis. American Journal Of Psychiatry, (164), 942-948. DOI: 10.1176/ajp.2007.164.6.942

Procuraduría Federal del Consumidor. (PROFECO, 2010) Salchichas para hot dog. El rey de los embutidos. El confesionario. Revista del Consumidor. Recuperado de https://www.gob.mx/cms/uploads/attachment/fil e/119149/Estudio_Salchichas_3445_Septiembre_2010.pdf 
Obesidad y sobrepeso en adolescentes, productos ultra procesados, alimentación, trastornos por déficit de la atención e hiperactividad.

Procuraduría Federal del Consumidor. (PROFECO, 2011) Cereales para niños. Revista del Consumidor. Recuperado de https://www.gob.mx/cms/uploads/attachment/fil e/119166/Estudio_Cereales_para_nin_os_3044_Abril_2011.pdf

Procuraduría Federal del Consumidor. (PROFECO, 2014) Estudio de Calidad Aderezos. Revista del Consumidor. Recuperado de https://www.gob.mx/cms/uploads/attachment/fil e/100416/42-

57RC444_Estudio_de_Calidad_Aderezos.pdf

Procuraduría Federal del Consumidor. (PROFECO, 2018) Frituras y botanas de maíz y trigo. Revista del Consumidor. Recuperado de https://www.gob.mx/cms/uploads/attachment/fil e/393470/Estudio_Calidad_Frituras_y_Botana.p df

Real Academia Española. (RAE, 2005) Fast Food. Diccionario Panhispánico de Dudas.

Sauceda G.J.M., Albores V., Capece J., Landeros E. y Martínez M.C. (2007) Impacto psicosocial del trastorno por déficit de atención con hiperactividad (TDAH). Psiquiatría, (23), 54.

Ulloa R.E., Sauceda J.M., Ortiz S. y Sánchez S. (2006) Psicopatología asociada al trastorno por déficit de atención e hiperactividad en niños de edad escolar. Actas Españolas de Psiquiatría, (34), 330-335.

Willcutt E. (2012) The Prevalence of DSM-IV Attention-Deficit/ Hyperactivity Disorder: A Meta-Analytic Review. Neurotherapeutics, (9), 490-499. DOI: $\underline{10.1007 / \mathrm{s} 13311-012-0135-8}$ 